\title{
Ontologia dos Objetivos Educacionais
}

\author{
Pablo R. F. de Oliveira ${ }^{1}$, Patrício de Alencar Silva ${ }^{2}$, Pedro Fernandes Ribeiro Neto ${ }^{3}$ \\ ${ }^{1}$ Universidade Federal Rural do Semi-Árido (UFERSA) e Universidade do Estado do Rio \\ Grande do Norte (UERN) \\ ${ }^{2}$ Universidade Federal Rural do Semi-Árido (UFERSA) \\ ${ }^{3}$ Universidade do Estado do Rio Grande do Norte (UERN) \\ \{pablorobertofernando, patricioalencar, pedro.fernandes2503\}@gmail. com
}

\begin{abstract}
This paper presents the development and application of an ontology for learning objects. The domain of ontology consists in the classification of educational goals, representing the concept of the Bloom Taxonomy. For the development of the same, the methodology of the guide 101 that offers the steps for an ontology construction was used, besides of tool Protegé. The ontology was validated through a hypothetical scenario and its integration with the K-hunters tool to assess its application potential. As a result, the correct classification of learning objects and users of the K-hunters tool was observed, through ontology.
\end{abstract}

Resumo. Este artigo apresenta o desenvolvimento e aplicação de uma ontologia para objetos de aprendizagem. $O$ domínio da ontologia considera a classificação dos objetivos educacionais, representando o conceito da Taxonomia de Bloom. Para o desenvolvimento do mesmo, foi utilizada a metodologia do guia 101, que oferece as etapas para a construção de uma ontologia, além da ferramenta Protegé. A ontologia foi validada por meio de um cenário hipotético e através de sua integração à ferramenta K-hunters para avaliar seu potencial de aplicação. Como resultado, foi observada a correta classificação dos objetos de aprendizagem e usuários da ferramenta K-hunters, através da ontologia.

\section{Introdução}

Ambientes virtuais de aprendizagem possibilitam a utilização de vários recursos educacionais que auxiliam o processo de ensino e aprendizagem, a exemplo dos objetos de aprendizagem, uma entidade digital que é utilizada para fins educativos [Wiley 2001]. São exemplos de objetos de aprendizagem: textos, imagens, áudio, vídeo, entre outros. O caráter pedagógico dos objetos de aprendizagem é essencial nestes ambientes e devem estar alinhados aos objetivos educacionais estabelecidos pelo professor.

Todavia gerenciar objetos de aprendizagem em ambientes virtuais que estejam alinhados aos objetivos educacionais exige atenção, como também avaliar o rendimento de alunos nesses ambientes, tendo em vista a necessidade de alinhar os objetivos estabelecidos dos objetos com as atividades de avaliação.

Como alternativa teórica à essa problemática a Taxonomia de Bloom [Bloom et al 1956] pode ser utilizada, pois organiza de maneira hierárquica os objetivos educacionais para 
auxiliar o processo de avaliação das habilidades dos alunos em três domínios: cognitivo, afetivo e psicomotor. E para criar e classificar objetos de aprendizagem em ambientes virtuais de aprendizagem que estejam alinhados a esses objetivos pode-se utilizar ontologias, pois permitem definir formalmente a relação entre termos e objetos, como no caso da Taxonomia de Bloom e os objetos de aprendizagem.

Uma ontologia consiste numa estrutura de conhecimento que define formalmente a relação entre termos de entidades digitais [Tim Berners-Lee 2001; Kück 2004) e por se tratar também de um artefato de software, o conhecimento contido em uma ontologia pode ser aproveitado por um sistema computacional e agentes inteligentes [Raju e Ahmed 2012]. Alguns repositórios de objetos de aprendizagem, ambientes virtuais de aprendizagem e jogos educacionais, por exemplo, utilizam ontologias para modelar ou classificar o aprendizado dos alunos [Botelho e Pires, 2008]. Assim, cadastrar e gerenciar de forma eficiente objetos de aprendizagem em um sistema, classificá-los em um dos níveis da Taxonomia de Bloom, como também acompanhar o progresso do aluno, é um processo que pode ser otimizado com a utilização de uma ontologia.

O estudo apresentado neste artigo teve como objetivo desenvolver uma ontologia de domínio para classificação de objetos de aprendizagem, considerando a hierarquia dos objetivos educacionais do domínio cognitivo da Taxonomia de Bloom. Como validação, a mesma foi integrada a um jogo educacional digital que utilizava um repositório de objetos de aprendizagem como principal recurso para promover a aprendizagem no ambiente virtual.

\section{Taxonomia de Bloom}

A taxonomia proposta por Bloom (et al. 1956), conhecida como taxonomia de Bloom, tem como objetivo ajudar no planejamento, organização e controle dos objetivos de aprendizagem.

Os objetivos educacionais são divididos pela taxonomia em três domínios: cognitivo, afetivo e psicomotor. O domínio cognitivo abrange a aprendizagem intelectual, o domínio afetivo abrange aspectos relacionados a sensibilização e gradação de valores pelo aluno, e o domínio psicomotor abrange as habilidades para a execução de tarefas que exigem o aparelho motor. A organização desses objetivos é hierárquica, partindo do objetivo mais simples até o mais complexo, proporcionando o desenvolvimento de habilidades que vão crescendo em complexidade até atingir os níveis mais altos [Lima 2009]. Dos domínios mencionados, o cognitivo tem sido um dos mais aplicados por ter sido implementado em sua totalidade.

A organização dos objetivos educacionais também é feita através de verbos que descrevem o processo de progressão cognitiva, expressa por meio de uma ação ou comportamento do aprendiz sobre o conteúdo a ser estudado. O domínio cognitivo envolve a aquisição de um novo conhecimento e agrupa os objetivos - verbos - em seis categorias ou níveis. Para ascender a uma nova categoria é preciso ter obtido um desempenho adequado na anterior. Cada categoria utiliza capacidades adquiridas nos níveis anteriores. As categorias do domínio cognitivo são: Conhecimento, Compreensão, Aplicação, Análise, Síntese e Avaliação [Ferraz; Belhot 2010]. A Figura 1 ilustra os níveis do domínio cognitivo da taxonomia.

Lima (2009) utilizou em seu trabalho a taxonomia de Bloom e propôs duas ferramentas pedagógicas para planejamento de cursos em ambientes virtuais de aprendizagem, o Mapa de Conteúdo e o Mapa de Dependência. As ferramentas foram 
IX Congresso Brasileiro de Informática na Educação (CBIE 2020)

Anais do XXXI Simpósio Brasileiro de Informática na Educação (SBIE 2020)

desenvolvidas para auxiliar o professor na escolha dos objetivos educacionais e nos verbos para os conteúdos do curso, conforme a taxonomia de Bloom em seu domínio cognitivo. O Quadro 1 apresenta respectivamente o comportamento do objetivo educacional e o verbo sugerido para estar presentes nos enunciados de atividades.

Quadro 1: Objetivos educacionais e seus respectivos verbos [Lima 2009, adaptado]

\begin{tabular}{|c|c|}
\hline Níveis & Comportamento/Verbos \\
\hline \multirow[b]{2}{*}{ Conhecimento } & $\begin{array}{l}\text { O aluno deverá recordar ou reconhecer informações, ideias e princípios na forma } \\
\text { idêntica ao que foi apresentado }\end{array}$ \\
\hline & $\begin{array}{l}\text { escreva, liste, mostre, tabule, enumere, copie, selecione, defina, reproduza, } \\
\text { identifique, cite... }\end{array}$ \\
\hline \multirow[b]{2}{*}{ Compreensão } & $\begin{array}{l}\text { O aluno deverá traduzir, compreender ou interpretar informações com base no que } \\
\text { foi apresentado. }\end{array}$ \\
\hline & $\begin{array}{l}\text { explique, associe, diferencie, estimule, agrupe, sumarize, converta, discuta, } \\
\text { traduza, ordene... }\end{array}$ \\
\hline \multirow[b]{2}{*}{ Aplicação } & $\begin{array}{l}\text { O aluno deverá usar um conhecimento anterior para resolver situação que não } \\
\text { tenham sido apresentadas a ele. }\end{array}$ \\
\hline & $\begin{array}{l}\text { use, compute, resolva, aplique, termine, experimente, demonstre, descubra, } \\
\text { prepare... }\end{array}$ \\
\hline \multirow[b]{2}{*}{ Análise } & $\begin{array}{l}\text { O aluno deverá distinguir, classificar e relacionar pressupostos, hipóteses, } \\
\text { evidências de um conteúdo apresentado. }\end{array}$ \\
\hline & $\begin{array}{l}\text { analise, classifique, categorize, compare, deduza, arranje, conecte, divida, } \\
\text { indique... }\end{array}$ \\
\hline \multirow[b]{2}{*}{ Síntese } & $\begin{array}{l}\text { O aluno deverá criar, integrar ou combinar ideias num produto, plano ou proposta } \\
\text { novos para ele. }\end{array}$ \\
\hline & $\begin{array}{l}\text { crie, proponha, formule, modifique, planeje, elabore, invente, projete, desenvolva, } \\
\text { adapte... }\end{array}$ \\
\hline \multirow[b]{2}{*}{ Avaliação } & $\begin{array}{l}\text { O aluno deverá apreciar, avaliar ou criticar algo com base em critérios e padrões } \\
\text { específicos. }\end{array}$ \\
\hline & $\begin{array}{l}\text { julgue, argumente, avalie, recomende, critique, justifique, decida, teste, convença, } \\
\text { conclua... }\end{array}$ \\
\hline
\end{tabular}

Como observado no Quadro 1, primeiramente define-se o objetivo de aprendizagem e posteriormente utiliza-se os verbos daquele objetivo em enunciados de atividades. Em ambientes virtuais essas atividades podem se apresentar em forma de objetos de aprendizagem, que também podem apresentar esses objetivos.

Desta forma a taxonomia de Bloom pode ser representada por uma ontologia, principalmente por se tratar de uma estrutura hierárquica. Assim como, objetos de aprendizagem notados semanticamente de metadados podem ser classificados por meio de uma ontologia em ambientes virtuais de aprendizagem.

\section{Metodologia}

Para esta pesquisa, utilizou-se a metodologia do guia 101 [Noy et al. 2001], que utiliza passos iterativos para a construção de uma ontologia.

Para a formalização da ontologia a linguagem utilizada foi a OWL (Ontology Web Language), pois estende o vocabulário de esquemas RDF (Resource Description Framework) ao incluir construtores mais ricos em relação à expressividade e à inferência [Bechhofer et al. 2004]. Essa etapa foi executada utilizando a ferramenta Protégé (2017), uma ferramenta dedicada à construção de ontologias. A escolha por esta ferramenta se deu 
pela possibilidade de realizar verificações na ontologia por meio dos motores de inferência que possui, como o $\mathrm{FaCT}++$.

O domínio desta ontologia concentra-se no aspecto Cognitivo da Taxonomia de Bloom, Quadro 1. Seu escopo consiste na classificação de objetos de aprendizagem, considerando as características dos mesmos: comportamento e verbo. Além de verificar a dependência entre os objetos de aprendizagem, conforme os níveis da taxonomia. Uma vez classificado, o objeto de aprendizagem possui propriedades que permitem inferir o nível cognitivo de uma instância que se relacione com o mesmo.

Como contexto de utilização da ontologia, tem-se os ambientes virtuais de aprendizagem, pois utilizam objetos de aprendizagem para auxiliar o aluno a alcançar objetivos educacionais. O jogo educativo K-hunters [Silva 2018] foi utilizado na validação da ontologia deste trabalho, pois utiliza objetos de aprendizagem em um contexto de aprendizagem virtual. O principal objetivo do jogo é auxiliar a aprendizagem de crianças com ou sem autismo. No jogo o aluno aprende através de objetos de aprendizagem que apresentam um questionário após a apresentação do conteúdo do objeto.

\section{Ontologia dos Objetivos Educacionais}

Como principal fonte para o levantamento dos termos da ontologia considerou-se a própria Taxonomia de Bloom, além do Mapa de Conteúdos e Mapa de Dependência [Lima 2009] que apresenta a construção de conteúdos virtuais a partir da definição dos objetivos educacionais. O Mapa de Conteúdo e o Mapa de Dependência são ferramentas pedagógicas que auxiliam na construção de cursos virtuais, utilizando a Taxonomia de Bloom. Para tanto, essas ferramentas disponibilizam comportamentos e verbos que irão compor determinado conteúdo. De maneira única, cada verbo e comportamento pertence a um conjunto de verbos e comportamentos, e cada um desses conjuntos pertencem a um dos níveis da taxonomia (Quadro 1).

Desta forma identificou-se termos que pertencem a um conjunto de Comportamentos \{comportamento1, ..., comportamento6\} e Verbos $\{$ verbo1, ..., verbo6\}. Como já mencionado, cada conjunto de comportamento e verbo pertence a um nível da taxonomia, que são: Conhecimento, Compreensão, Aplicação, Análise, Síntese e Avaliação. Cada nível da taxonomia possui um comportamento a ser desempenhado pelo aluno e um verbo que pode estar presente no conteúdo ou em alguma atividade. Todavia, os níveis da taxonomia são hierárquicos, portanto ainda percebeu-se a necessidade do seguinte conjunto de termos: Nivel\{nível1, ..., nivel6\}, para organizar os objetos de aprendizagem conforme a dependência hierárquica dos mesmos.

\subsection{Classes}

As classes são a organização dos conceitos de um domínio em hierarquias. A ontologia desenvolvida possui 30 classes, sendo 5 classes gerais: MapaDeConteudo, MapaDeDependencia, ObjetivoEducacional e TAXONOMIA. A classe MapaDeDependencia possui 6 subclasses, considerando os seis níveis do domínio cognitivo da taxonomia, a classe ObjetivoEducacional reúne duas classes gerais Comportamento e Verbo, que possuem 6 subclasses cada uma: comportamento 1, ..., comportamento6; e verbo1, ..., verbo6. Cada subclasse de Comportamento e Verbo possui verbos e comportamentos distintos, conforme cada nível da taxonomia. Por fim, a classe TAXONOMIA possui subclasses relacionadas aos 6 níveis da taxonomia: Conhecimento, Compreensão, Aplicação, 
Análise, Síntese e Avaliação. Esta última classe é responsável por classificar um indivíduo/instância em algum nível da Taxonomia de Bloom.

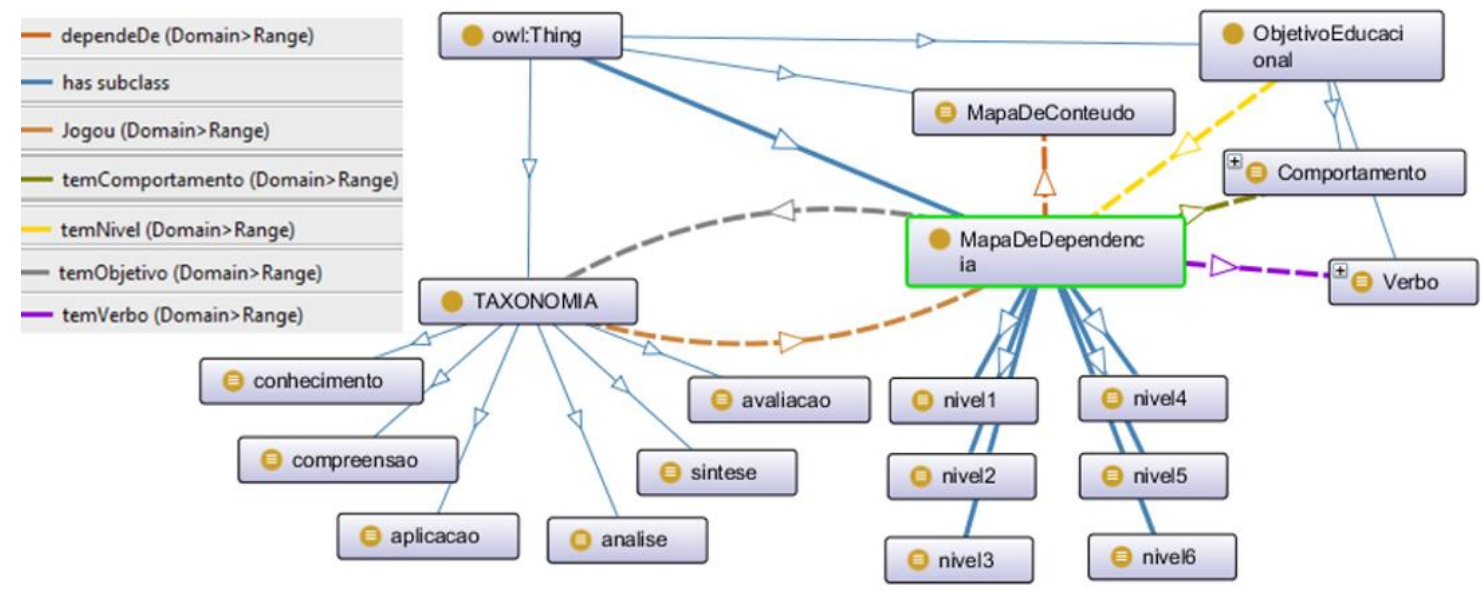

Figura 1: Visualização das Classes da Ontologia na Ferramenta Protégé.

Classes ainda podem ser consideradas primitivas ou definidas. Caso uma classe tenha apenas condições necessárias, ela é conhecida como uma classe primitiva. As classes definidas podem classificar as classes definidas, classes primitivas e indivíduos. A Figura 1 apresenta as classes primitivas da ontologia. O Quadro 2 apresenta as classes definidas da ontologia deste trabalho.

Quadro 2: Classes definidas da Ontologia

\begin{tabular}{|l|l|}
\hline \multicolumn{1}{|c|}{ Classes } & \multicolumn{1}{|c|}{ Representação } \\
\hline $\begin{array}{l}\text { nivell, nivel2, nivel 3, nivel4, } \\
\text { nivel5, nivel6. }\end{array}$ & $\begin{array}{l}\text { Representam a dependência de cada nível da Taxonomia de Bloom, } \\
\text { respectivamente, e classifica as instâncias de objetos de } \\
\text { aprendizagem. }\end{array}$ \\
\hline $\begin{array}{l}\text { conhecimento, compreensao, } \\
\text { aplicacao, analise, sintese, } \\
\text { avaliacao. }\end{array}$ & $\begin{array}{l}\text { Representam cada nível da Taxonomia de Bloom e classifica os } \\
\text { indivíduos que se relacionam com alguma instância que representa } \\
\text { umobjeto de aprendizagem deste nível. }\end{array}$ \\
\hline
\end{tabular}

A Listagem 1 apresenta o axioma da classe nivel 2 e classifica indivíduos que possuem um valor de conteúdo, um verbo e um comportamento. Além disso os indivíduos classificados através deste axioma também possuem uma dependência com alguma instância do seu nível anterior. Instância desse tipo são os objetos de aprendizagem, que possuem para o contexto da aplicação desta ontologia: um verbo, um comportamento, um valor de conteúdo e a dependência de outro objeto.

Listagem 1: Axioma Mapa de Dependência, classe nível2

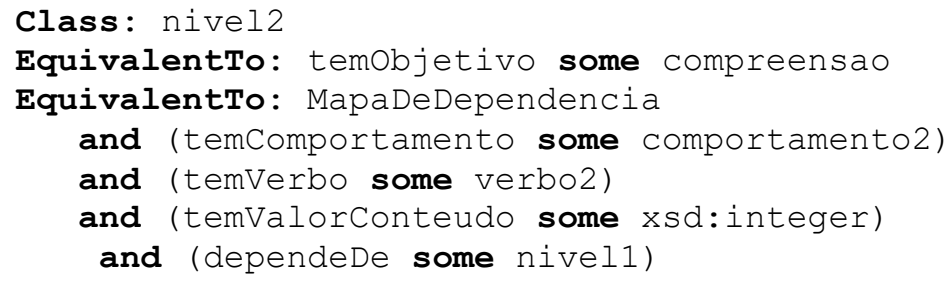


Listagem 2: Axioma TAXIONOMIA, classe compreesao

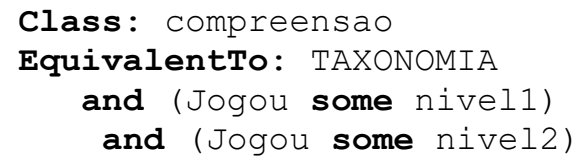

Já a Listagem 2 diz respeito a classificação de indivíduos do tipo aluno e apresenta o axioma da classe compreensão, do nível 2 da Taxonomia de Bloom. Para ser definido nesta classe o indivíduo deve ter se relacionado com 2 níveis da taxonomia através da propriedade Jogou. Ou seja, o indivíduo se relacionou com instâncias definidas nos níveis do 1 ao 2. Para o contexto de aplicação desta ontologia, esses indivíduos são os alunos que utilizam objetos de aprendizagem.

O relacionamento entre classes se dá por meio de propriedades. As propriedades de uma classe são herdadas por suas subclasses. A seção a seguir apresenta as propriedades da ontologia deste trabalho.

\subsection{Propriedades das Classes}

A presente ontologia possui seis Object Properties. Primeiramente a propriedade dependeDe permite relacionar indivíduos da classe MapaDeDependencia com indivíduos da classe TAXONOMIA, ou seja, indivíduos da classe TAXONOMIA, como um objeto de aprendizagem, com indivíduos da classe MapaDeDependencia. A propriedade temNivel relaciona as classes TAXONOMIA e MapaDeDependencia, permitindo o relacionamento de indivíduo aluno, por exemplo, com alguma instância de MapaDeDependencia. Essas duas primeiras propriedades são do tipo funcional, logo, um indivíduo 'a' pode relacionar-se a exatamente um indivíduo ' $b$ ' a partir da propriedade.

As propriedades temVerbo e temComportamento relacionam as classes Verbo e Comportamento com a classe MapaDeDependencia. São do tipo transitiva e permite que uma instância do tipo objeto de aprendizagem se relacione com instâncias do tipo verbo e comportamento, classificando em um dos níveis do MapaDeDependencia. Por fim, a propriedade jogou, relaciona uma instância do tipo aluno com a classe TAXONOMIA de forma transitiva. Essas três propriedades são subclasses de temObjetivo que relaciona TAXONOMIA com MapaDeDependencia.

Foram também utilizadas propriedades de dados para auxiliar no mapeamento da Ontologia. A propriedade do tipo de dados (Data Property) utiliza uma variável para representar qualquer coisa no domínio abordado. É necessário definir o domínio a qual ela pertence e o seu valor que será um elemento do tipo string, boolean, int, por exemplo. As propriedades de dados da ontologia proposta são temValorConteudo e temValorID, ambas do tipo $x$ sd:integer.

As propriedades de dados permitem que algumas informações possam ser armazenadas na ontologia, como o valor do conteúdo e o id do usuário. Como restrição têmse para as duas propriedades a característica funcional, logo, a propriedade impede que um indivíduo tenha mais de um valor de conteúdo, ou valor de id.

\subsection{Instâncias da Ontologia}

As instâncias também podem ser chamadas de indivíduos. Para a ontologia deste trabalho algumas instâncias foram inseridas a fim de auxiliar na classificação dos indivíduos do tipo 
objetos de aprendizagem. Atualmente a ontologia conta com aproximadamente cem instâncias que sempre permanecem na ontologia. Essas instâncias estão alocadas às subclasses das classes MapaDeDependência e ObjetivoEducacional, e são os níveis da taxonomia, verbos e comportamentos. A maioria das instâncias permanentes da ontologia são verbos e comportamentos presentes na Taxonomia de Bloom. Cada verbo e comportamento pertence a uma única classe - subclasses de comportamento ou verbo.

\section{Validação da Ontologia}

A validação da ontologia aconteceu a partir da verificação da sua corretude, conferindo se a mesma se comporta de forma correta para o domínio que representa. Através de um cenário hipotético a verificação analisou a completude e consistência da ontologia.

Utilizou-se para verificar a ontologia, o motor de inferência reasoner $\mathrm{FaCT}++$, presente na ferramenta Protégé. A Figura 2 (a) apresenta o resultado da inferência para a instância 'Figura'. A instância se relaciona com as instâncias 'distinga', 'compreender' e 'Podcast'. Além disso ela possui o valor do 'Conteúdo1'. O resultado da inferência é a classificação da instância 'Figura' na sua subclasse nivel2. 'Figura' representa um objeto de aprendizagem que possui o verbo distinga e o comportamento compreender. A classe definida nivel 2 possui o axioma que permite a classificação correta no nível do verbo e comportamento do objeto, além de verificar se o mesmo depende de um nível anterior. Com relação ao nível anterior, a instância 'Podcast' foi classificada no nivell, possibilitando a classificação correta de 'Figura'.

\begin{tabular}{|c|c|c|c|}
\hline Description: Figura & Property assertions: Figura & Description: Maria & Property assertions: Maria \\
\hline $\begin{array}{l}\text { Types } † \\
\text { @ nivel2 } \\
\text { Same Individual As } † \\
\text { Different Individuals } †\end{array}$ & $\begin{array}{l}\text { Object property assertions } \oplus \\
\text { dependeDe Podcast } \\
\text { temComportamento compreender } \\
\text { temVerbo distinga } \\
\text { temObjetivo compreender } \\
\text { temObjetivo distinga } \\
\text { Data property assertions } \oplus \\
\text { temValorConteudo } \mathbf{1}\end{array}$ & 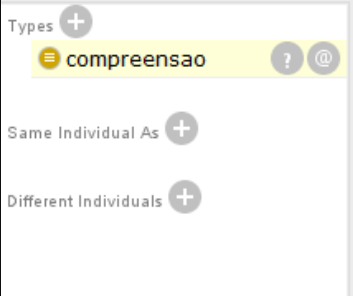 & $\begin{array}{l}\text { Object property assertions }+ \\
\text { Jogou Podcast } \\
\text { Jogou Figura } \\
\text { temObjetivo Podcast } \\
\text { temobjetivo Figura } \\
\text { Data property assertions } \$ \\
\text { temValorID } 1\end{array}$ \\
\hline
\end{tabular}

(a)

(b)

Figura 2: (a) Inferência para um objeto de Aprendizagem. (b) Inferência para um aluno.

A Figura 2 (b) apresenta o resultado da inferência para a instância 'Maria' que é classificada como sendo do tipo conhecimento. A instância se relaciona com duas outras instâncias: 'Podcast' e 'Figura'. 'Maria' representa um aluno, pois se relaciona com as demais instâncias através da propriedade Jogou, além de possuir um valor de identificação.

A classe definida compreensao reúne instâncias que se relacionam com outras instâncias do tipo nivell e nivel2. Para ser classificado em compreensao, o indivíduo deverá estar relacionado a duas instâncias, uma do nivell e outra do nivel2. De acordo com a Taxonomia de Bloom, os níveis cognitivos são hierárquicos, logo, para se alcançar um nível posterior, deve-se adquirir o conhecimento do nível atual. Neste exemplo hipotético 'Maria' precisou ser classificada primeiramente em conhecimento, por estar relacionada a 'Podcast' que é do tipo nívell, para então ser classificada em compreensao, considerando o relacionamento com a instância 'Figura' do tipo nivel2.

\subsection{Verificação da Ontologia}

O jogo K-Hunters faz parte de um projeto desenvolvido e mantido através da parceria entre a Universidade Federal Rural do Semi-Árido (UFERSA) e a Unidade de Ensino Superior 
Dom Bosco (UNDB). O objetivo do projeto e consequentemente do jogo é minimizar o isolamento de crianças com espectro autista (TEA), além de possibilitar aprendizagem através dos objetos de aprendizagem.

A aplicação se comunica com o repositório de objetos de aprendizagem buscando os objetos nele contidos. O especialista insere as informações que compõem os objetos de aprendizagem nos repositórios, carrega o objeto de aprendizagem e em seguida escolhe o monstro a que estará associado o objeto de aprendizagem e a sua localização. $\mathrm{O}$ especialista também pode inserir alguma pergunta sobre o conteúdo do objeto de aprendizagem.

A ontologia dos objetivos de aprendizagem deste trabalho foi integrada à ferramenta K-hunters nesta pesquisa para verificar a utilidade da ontologia dos objetos de aprendizagem em um contexto virtual de aprendizagem. Para tanto, algumas funcionalidades foram adicionadas à ferramenta. Outrossim, além de classificar os objetos, a ontologia também permitiu a classificação das crianças em uma das categorias do domínio cognitivo da taxonomia, considerando o objeto de aprendizagem acessado e a avaliação.

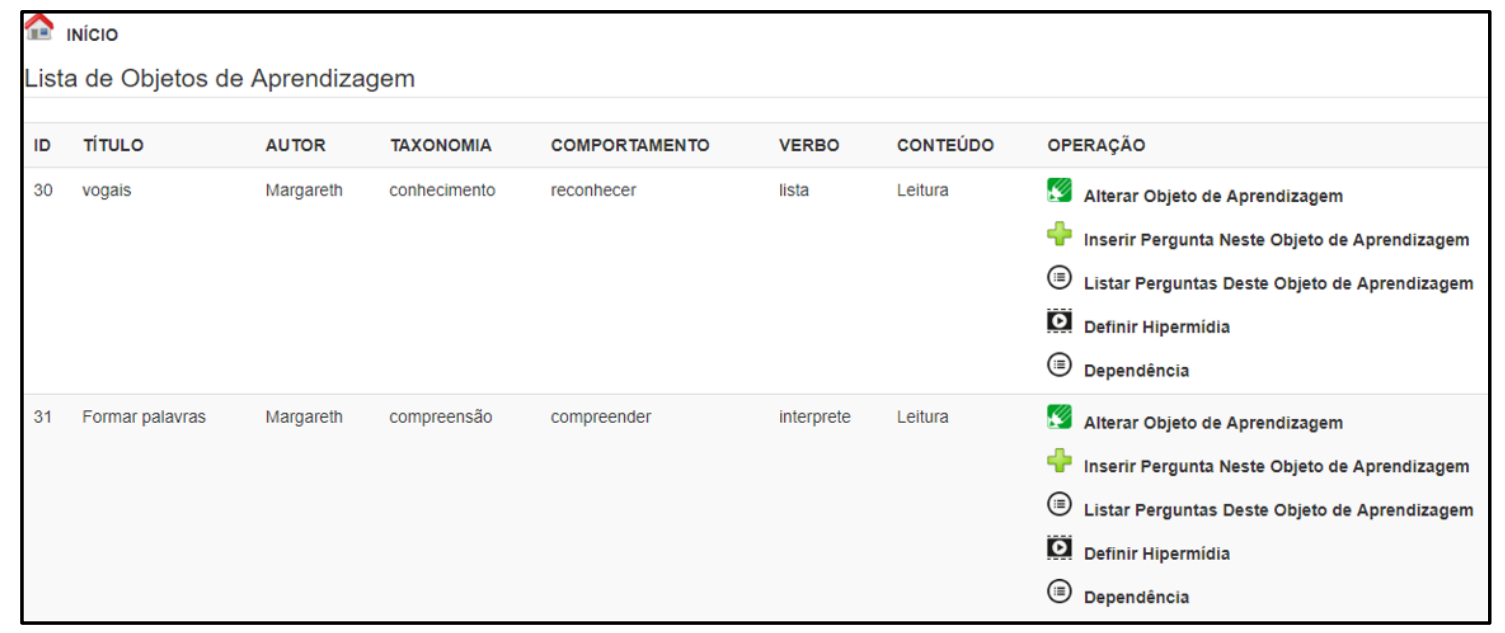

Figura 3: Lista dos objetos de aprendizagem cadastrados e classificados.

No momento do cadastro do objeto de aprendizagem na ferramenta, o professor insere um comportamento e um verbo que estará presente na atividade a ser desenvolvida pelo aluno. Depois deve informar a dependência com outro objeto de um nível anterior, caso exista. A partir da base de conhecimento ontológica e de um Sistema Multiagente (SMA) o objeto cadastrado é classificado em um dos níveis de dependência da ontologia, considerando o objeto de aprendizagem de quem ele depende, como mostra a Figura 3, na informação 'TAXONOMIA'. O objeto de ID 30 foi classificado no nível conhecimento, considerando que não havia dependência como outro objeto e o verbo e comportamento do nível 1 da taxonomia. Já o objeto de ID 31 foi classificado no nível compreensão, pois havia uma dependênicia estabelecida com um objeto do nível 1 (ID 30), além do verbo e comportamento do nível 2 da taxonomia.

Após cadastrar um objeto de aprendizagem o professor pode inserir uma questão relacionada ao mesmo. Para essa questão são considerados o verbo que o professor cadastrou no objeto. Essa informação é importante para classificar o aluno. A Figura 4 apresenta a classificação de um aluno para um conteúdo (Conteúdo Leitura). Para este conteúdo foram cadastrados 6 objetos de aprendizagem e perguntas associadas aos mesmos. Como pode-se observar o aluno é classificado na medida que responde de maneira correta uma atividade 
IX Congresso Brasileiro de Informática na Educação (CBIE 2020)

Anais do XXXI Simpósio Brasileiro de Informática na Educação (SBIE 2020)

associada a um objeto de aprendizagem já classificado, como também se obteve êxito em uma atividade de um nível anterior, considerando a dependência.

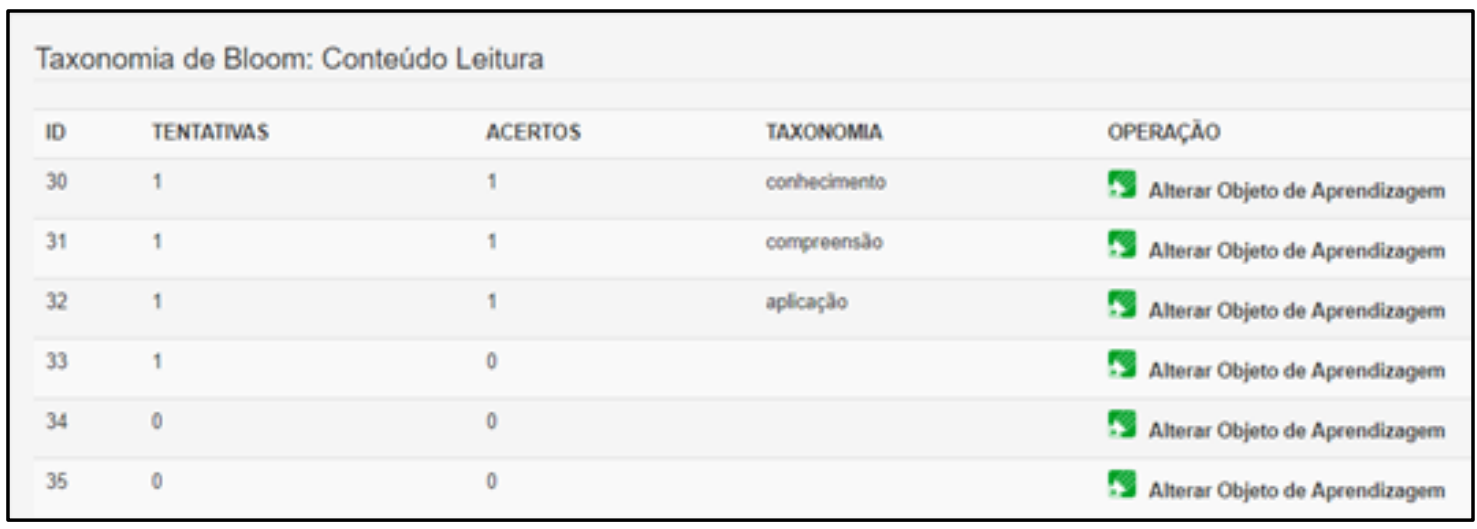

Figura 4: Classificação do aluno para um Conteúdo.

Na classificação do objeto de aprendizagem, um agente do SMA insere os dados na ontologia local para classificar o aluno. Para tanto, primeiramente o agente verifica a dependência do objeto de aprendizagem que o usuário utilizou, em seguida consulta na ontologia se o usuário também jogou o objeto da dependência, por fim obtém-se a classificação do aluno em um dos níveis da taxonomia (Figura 4).

\section{Considerações Finais}

A pesquisa demonstrou que os objetos de aprendizagem podem ser mapeados em níveis a partir das suas propriedades: comportamento e verbos. Bem como, considerando que cada verbo e comportamento possuem uma classificação taxonômica única, pôde-se definir a dependência destes objetos. Também foi possível classificar um aluno em um dos níveis cognitivos da Taxonomia de Bloom, a partir da relação da instância do usuário com a instância do objeto de aprendizagem.

O contexto de uso em que a ontologia foi aplicada demonstrou o potencial de aplicação que a mesma possui. Tendo em vista que o cadastro dos verbos e comportamentos inseridos para cada objeto cadastrado na ferramenta K-hunters, bem como a dependência dos mesmos, permitiu classificar os objetos de aprendizagem em níveis de dependência e a classificar os seus usuários em um dos níveis cognitivos da Taxonomia de Bloom.

\section{Referências}

Bechhofer, S. et al. Web Ontology Language (OWL), W3C recommendation, 2004. Disponivel em: <http://www.w3.org/TR/owl-ref/>. Acesso em: Maio 2018.

Berners-Lee, T., James H., and Ora L. (2001) "The semantic web." Scientific american 284, no. 5 .

Bloom, B. S. et al. (1956), Taxonomy of educational objectives. New York: David Mckay. v. 1. p.262.

Botelho, R. P., and Pires, D. F. (2008). "Uso de ontologias para a representação semântica de objetos de aprendizagem." In Companion Proceedings of the XIV Brazilian Symposium on Multimedia and the Web (pp. 158-160). ACM. 
Ferraz, A. P. C. M.; Belhot, R. V. (2010), Taxonomia de Bloom: revisão teórica e apresentação das adequações do instrumento para definição de objetivos instrucionais. Gest. Prod., São Carlos, v. 17, n. 2, p. 421-431.

Kück, G. (2004) "Tim Berners-Lee's Semantic Web." South African Journal of Information Management 6, no. 1.

Lima, R. W. (2009), Mapa de Conteúdos e Mapa de Dependências: ferramentas pedagógicas para uma metodologia de planejamento baseada em objetivos educacionais e sua implementação em um ambiente virtual de aprendizagem. Tese (Doutorado em Engenharia Elétrica) - Universidade Federal do Rio Grande do Norte, Natal.

Noy, N. F.; Mcguinness, D. L. et al. (2001), Ontology development 101: A guide to creating your first ontology. [S.1.]: Stanford knowledge systems laboratory technical report KSL-01-05 and Stanford medical informatics technical report SMI-2001-0880, Stanford, CA.

Protégé. (2017), Stanford University School of Medicine. Disponível em: $<\underline{\text { http://protege.stanford.edu/> }}$

Raju, P; Ahmed, V. (2012) Enabling technologies for developing next-generation learning object repository for construction. Automation In Construction, [s.1.], v. 22, p.247-257, mar. 2012. Elsevier BV.

Silva, S. D. (20018) Knowledgemon Hunters: Um Jogo Sério com Geolocalização para Apoiar a Aprendizagem de Crianças com Autismo e Dificuldades de Aprendizado. 143 p. Dissertação (Programa de Pós-Graduação em Ciência da Computação) Universidade do Estado do Rio Grande do Norte, Universidade Federal Rural do Semi-Árido. 